\title{
Decompensation of chronic open-angle glaucoma following mydriasis-induced pigmentary dispersion into the aqueous humour: a light and electron microscopic study
}

\author{
RAJA HADDAD, ${ }^{1}$ GOTTFRIED STRASSER,${ }^{1}$ PETER HEILIG, ${ }^{1}$ \\ AND WOLFGANG JURECKA ${ }^{2}$
}

From the ${ }^{12 n d}$ Department of Ophthalmology and the 22nd Department of Dermatology, University of Vienna, Austria

SUMMARY A patient with well controlled chronic open-angle glaucoma developed after dilatation of both pupils persistent increase in intraocular pressure (IOP) due to extensive pigmentary dispersion into the aqueous humour. Trabeculectomy specimens obtained from both eyes after 3 and 7 weeks were studied by light and electron microscopy. It seems evident that the initial phase of raised IOP was caused by a clogging mechanism to the outflow channels by melanin and phagocytic cells. The permanent increase in IOP is attributed to the damage induced in the fibrous components of the trabecular sheets as a result of a complete breakdown of their endothelial covering.

Dilatation of the pupil occasionally results in an excessive release of pigment into the aqueous humour, sometimes associated with a transient increase in intraocular pressure (IOP). This phenomenon may occur in normal eyes of aged people on instillation of sympathomimetics ${ }^{1}$ but is frequently seen in glaucomatous eyes, especially if pigmentary dispersion and exfoliation syndromes are also present. $^{2-4}$ We report on a patient with well controlled primary open-angle glaucoma who, after dilatation of both pupils, developed a marked pigmentary dispersion and a nonresponsive increase in IOP requiring surgery in both eyes. Histological and electron microscopical examination of the trabeculectomy specimens obtained from both eyes disclosed changes that would explain the mechanism which led to the intractable rise in intraocular pressure.

\section{Case report}

An 83-year-old woman who was known to have chronic open-angle glaucoma with marked cupping of the optic discs and advanced bilateral visual field loss was finally treated with $0.25 \%$ timolol twice

Correspondence to Dr R. Haddad, 2nd Department of Ophthalmology, Alser Strasse 4, 1090 Vienna, Austria. daily maintaining an IOP of $18-20 \mathrm{mmHg}$ in both eyes. Moderate bilateral nuclear sclerosis and senile macular changes were also present. Best corrected visual acuity was RE 6/9 (20/30) and LE 6/60 (20/200). Otherwise, ocular examination showed no abnormality. The iris showed no transillumination, and there was no evidence of pigmentary glaucoma or of pseudoexfoliation syndrome. On routine pupillary dilatation with phenylephrine $(2.5 \%)$ (performed for fundus photography) a conspicuous release of pigment into the anterior chamber developed, with some aqueous flare and a marked deposition of pigment granules on the iris, lens capsule, and posterior surface of the cornea. Within 1 hour the IOP increased to $30 \mathrm{mmHg}$ in the right eye and to $34 \mathrm{mmHg}$ in the left eye. Gonioscopy revealed a massive accumulation of pigment on various structures of the angle, and the trabecular meshwork, mainly in the lower half of the globe, was densely pigmented. The patient was then treated with pilocarpine $(2 \%)$ and $250 \mathrm{mg}$ of acetazolamide intravenously. Two hours later the IOP was $29 \mathrm{mmHg}$ in the right eye and $30 \mathrm{mmHg}$ in the left eye. Pilocarpine was again given, but the IOP remained unaffected. During the following 3 weeks various drugs including timolol, carbachol, neostigmine, and tosmilen were tried, but no reduction in IOP could be achieved. There was, 

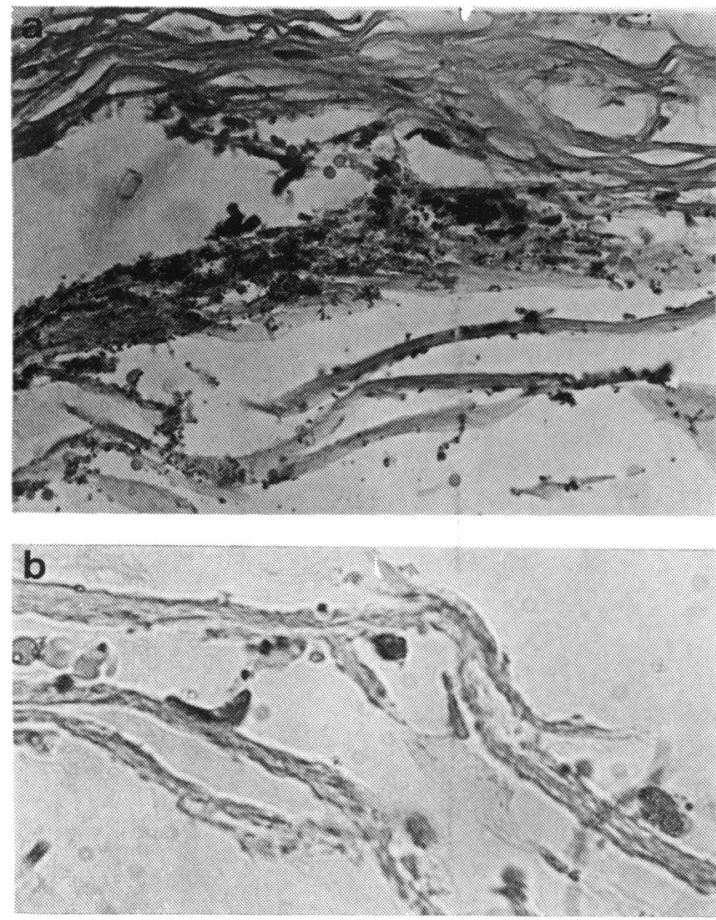

Fig. 1 Trabeculectomy specimen, left eye. (a) Melanin and pigment-laden cells clog the deep portion of the trabeculum. (b) Trabecular sheets are hyalinised and thickened with a few endothelial cells. $(\mathrm{a} \times 90 ; \mathrm{b} \times 217)$.

however, a continuous decrease in aqueous flare during this time, and the trabecular meshwork became noticeably less pigmented. At the end of the third week the IOP in both eyes was still uncontrolled, and trabeculectomy had to be performed first in the left eye and, 4 weeks later, in the right eye. On gonioscopic examination of the right eye prior to surgery most of the pigmentary deposits in this area had disappeared, and the trabecular meshwork was only slightly pigmented. Postoperatively the IOP in both eyes dropped to a normal level.

Trabeculectomy and iridectomy tissue obtained from the left eye was fixed in formalin and embedded in paraffin for light microscopy. The trabeculectomy specimen from the right eye was fixed in $3 \%$ glutaraldehyde and prepared for electron microscopy.

\section{Results}

Left eye. In the trabeculectomy specimen (Figs. 1 and 2) the trabecular meshwork was heavily pigmented, particularly in the deep portion. The trabe- cular sheets were thickened and hyalinised and had lost most of the endothelial cells (Fig. 1). The small number of the remaining endothelial cells appeared swollen, and a few of them contained melanin granules (Fig. 2). Within the intertrabecular spaces large amounts of pigment and melanin-laden cells were present. There was evidence that some of these cells were of endothelial origin and had sloughed off the trabecular beams. In the lumen of Schlemm's canal erythrocytes and some pigmentcontaining cells were present.

Iris. Both iris stroma and pigment epithelium showed no pathological changes.

Right eye. In the trabeculectomy specimen (Figs. $3-5)$ the most obvious findings were the severe
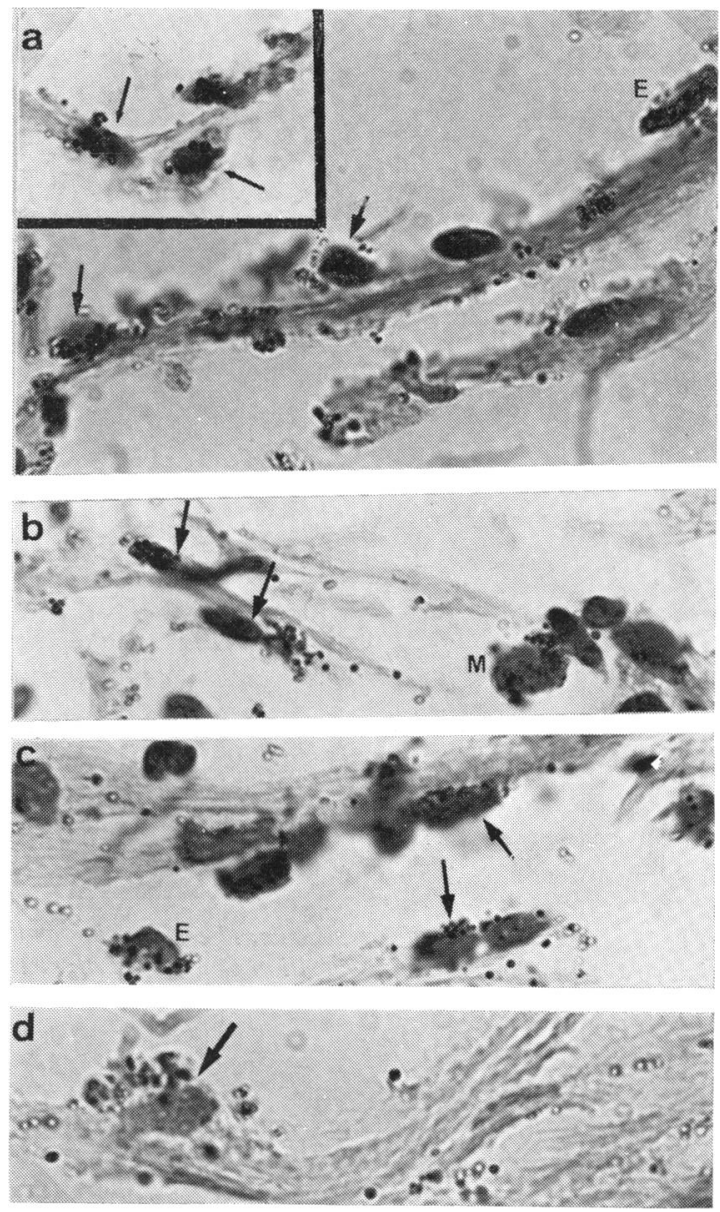

Fig. 2 Trabecular endothelium engulfing melanin (arrows in a, b, and c). Some cells appear to desquamate (E). Histiocytic macrophage (M). (d) Degenerated endothelial cell engorged with melanin (arrow). (a, b, c, $\times 283$; Inset $\times 227$; $\mathrm{d} \times 354$.) 


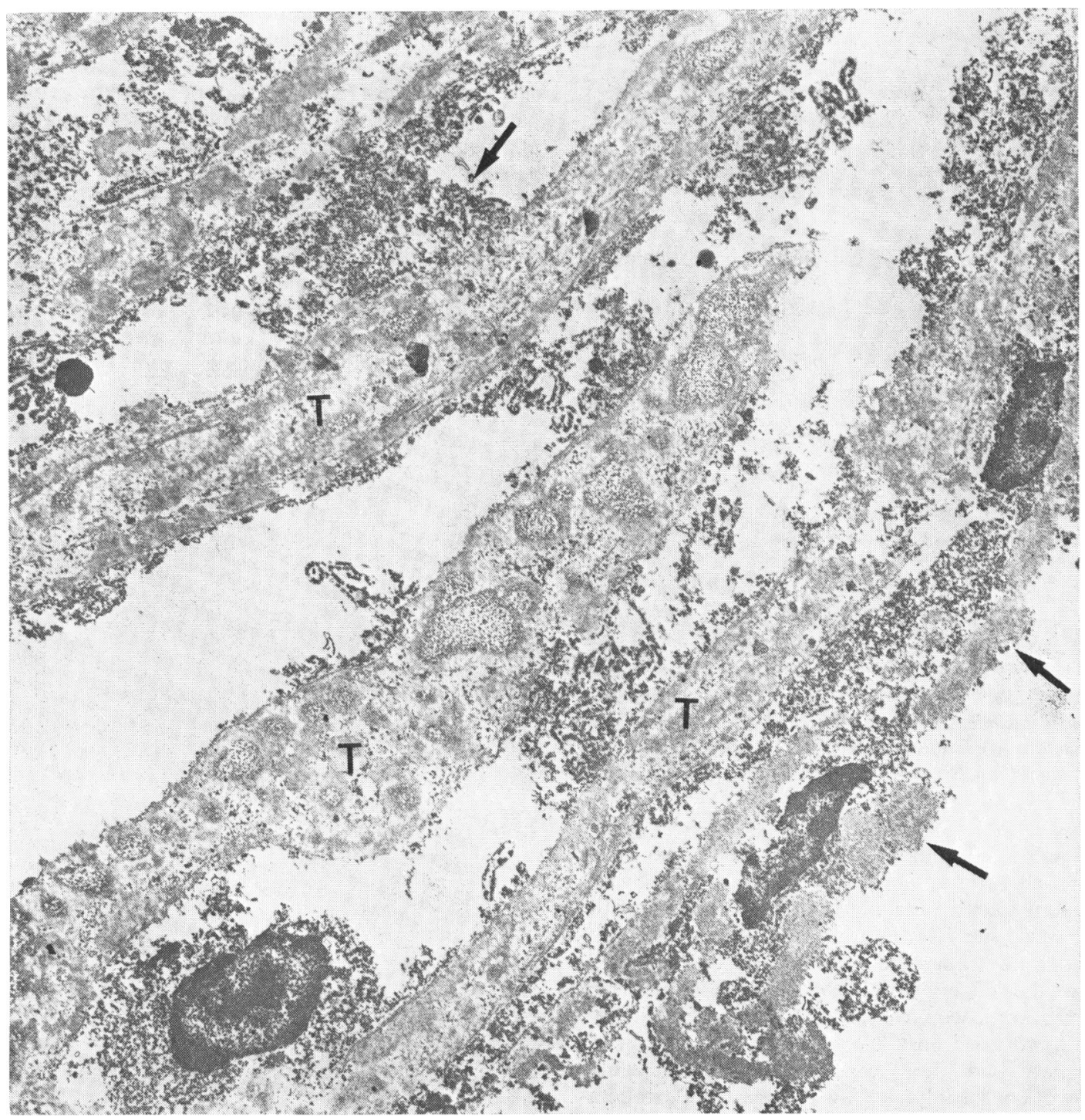

Fig. 3 Right eye. Swollen trabecular sheets lacking endothelial cells; few degenerating cells are seen. Narrowed intertrabecular spaces with deposition of material of varying density (arrows). $(\times 6000$.)

degeneration of the trabecular sheets, which were almost completely denuded of their endothelial covering, and marked intertrabecular densifications. Individual trabecular cords were swollen and covered by altered collagen, and occasional endothelial cells had degenerated (Figs. 3, 4). Only a few melanin granules and a small number of pigment-bearing cells were noted within the intertrabecular spaces. This is in sharp contrast to the large amount of melanin and macrophages in the specimen excised 4 weeks earlier from the other eye.

\section{Discussion}

Several studies have dealt with the passage of particulate material through the drainage angle, but only few reports concerned with the fate of pigment entering the trabecular area are available. 
It has long been known that melanin granules, liberated normally in eyes of elderly people, are subjected to phagocytosis by the cells of the trabecular meshwork..$^{5}$ This process of phagocytosis was also demonstrated under pathological conditions, where excessive release of pigment into the aqueous humour may occur. ${ }^{6}$ Histological studies of trabeculectomy specimens obtained from patients with pigmentary glaucoma show endothelial and histiocytic cells engulfing melanin granules that have accumulated in large amounts within the intertrabecular spaces. ${ }^{7-9}$ Native endothelial cells and
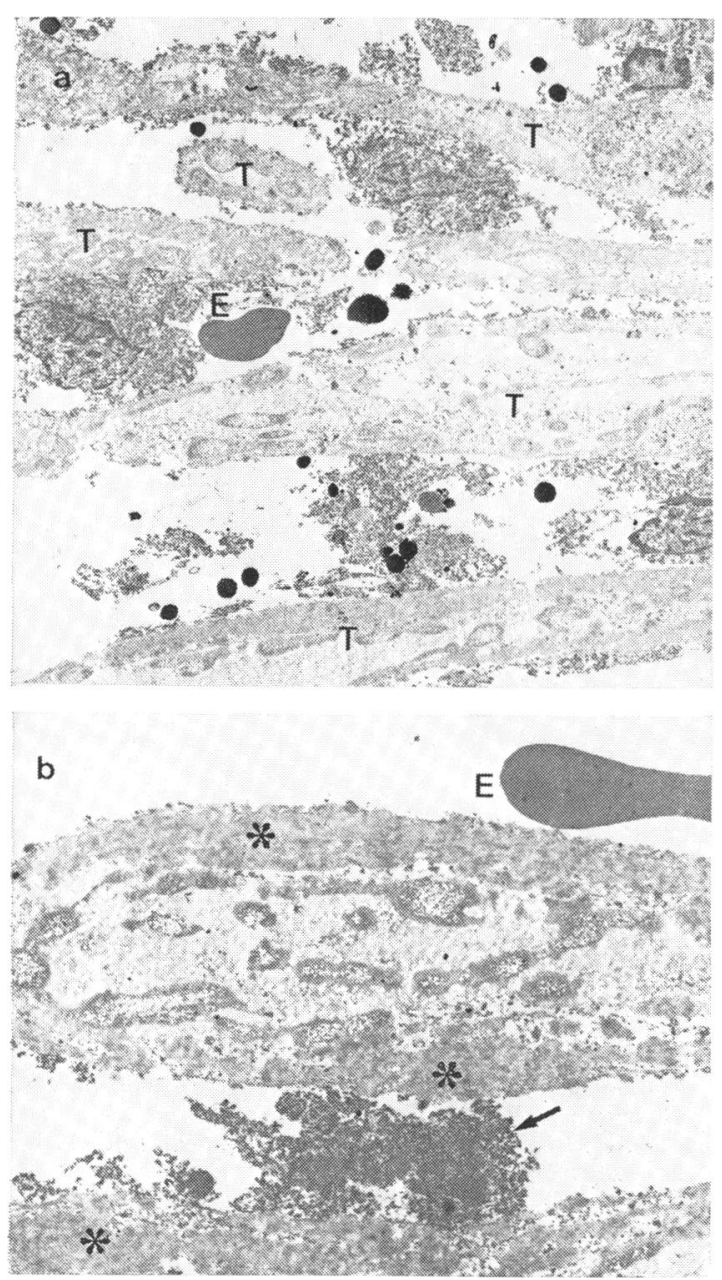

Fig. 4 (a) As in Fig. 3, with few pigment granules between the degenerating trabeculae $(\mathrm{T})$.

Erythrocyte $(\mathrm{E}) .(\times 3580$.) (b) Swollen trabecular sheet covered by altered collagenous material (asterisk). Degenerating material between individual beams (arrow). Erythrocyte (E). $(\times 7150$.) wandering histiocytes are known to play an important role in the disposal of various types of material from the drainage angle such as Indian ink, ${ }^{10}$ pseudoexfoliation material, ${ }^{11}$ bacteria and tapetum rods, ${ }^{12}$ erythrocytes, ${ }^{13}{ }^{14}$ and cellular debris. ${ }^{15}$ In a histological study of a trabeculectomy specimen from an eye with pigmentary glaucoma Richardson et al. ${ }^{9}$ found that, after phagocytosis, degeneration and desquamation of the trabecular endothelial cells had resulted in denudation and swelling of the trabecular sheets and collapse of the intertrabecular spaces. These observations are in agreement with those made by Rohen and Unger, ${ }^{16}$ Tripathi, ${ }^{17}$ and other workers, who have studied the trabecular meshwork following extensive phagocytosis of red blood cells ${ }^{18}$ and of injected foreign material. ${ }^{10}$

The presence of a large number of pigment-laden cells and of denuded and swollen trabecular sheets in the present case is apparently a result of extensive release of pigment, which has provoked a marked phagocytosis. Massive accumulation of pigment in the chamber angle, as is seen following mydriasis, may obstruct the outflow channels temporarily and cause a transient increase in IOP. ${ }^{2}$ Similarly, a large number of melanin-laden macrophages (from necrotic malignant uveal melanomas) collecting in this area were reported to account for the rise in IOP sometimes observed in these eyes. ${ }^{19}$ The blocking effect of macrophages on the aqueous outflow pathway has been demonstrated in other types of open-angle glaucoma. ${ }^{20-22}$ It is conceivable that the sudden increase in IOP in our patient was caused by a similar mechanical obstruction. However, the effect of such an obstruction seems to be valid only in the initial phase of the elevated IOP, since this remained unchanged, even after a marked decrease in the deposited pigment was noted on gonioscopy.

From a comparison of the morphological changes in the excised tissue 3 and 7 weeks following the acute release of pigment it becomes evident, furthermore, that the permanent increase in IOP in this patient was not a result of melanin or cellular blockage of the outflow channels. As already mentioned, it was evidently due to alterations in the fibrous components and swelling of the trabeculae after degeneration of its endothelium. It is difficult to say to what extent the pre-existing changes (hyalinisation of the sheets and intertrabecular densifications) attributed to chronic open-angle glaucoma ${ }^{23}$ have contributed to the final decompensation of IOP. It is very likely that the primary lesion lies within the 'subnormally functioning' endothelial cells which, on excessive phagocytic stimulation, ultimately broke down. ${ }^{24}$ The lack of the trabecular covering seems to have caused irreversible changes to the trabecular sheets and 


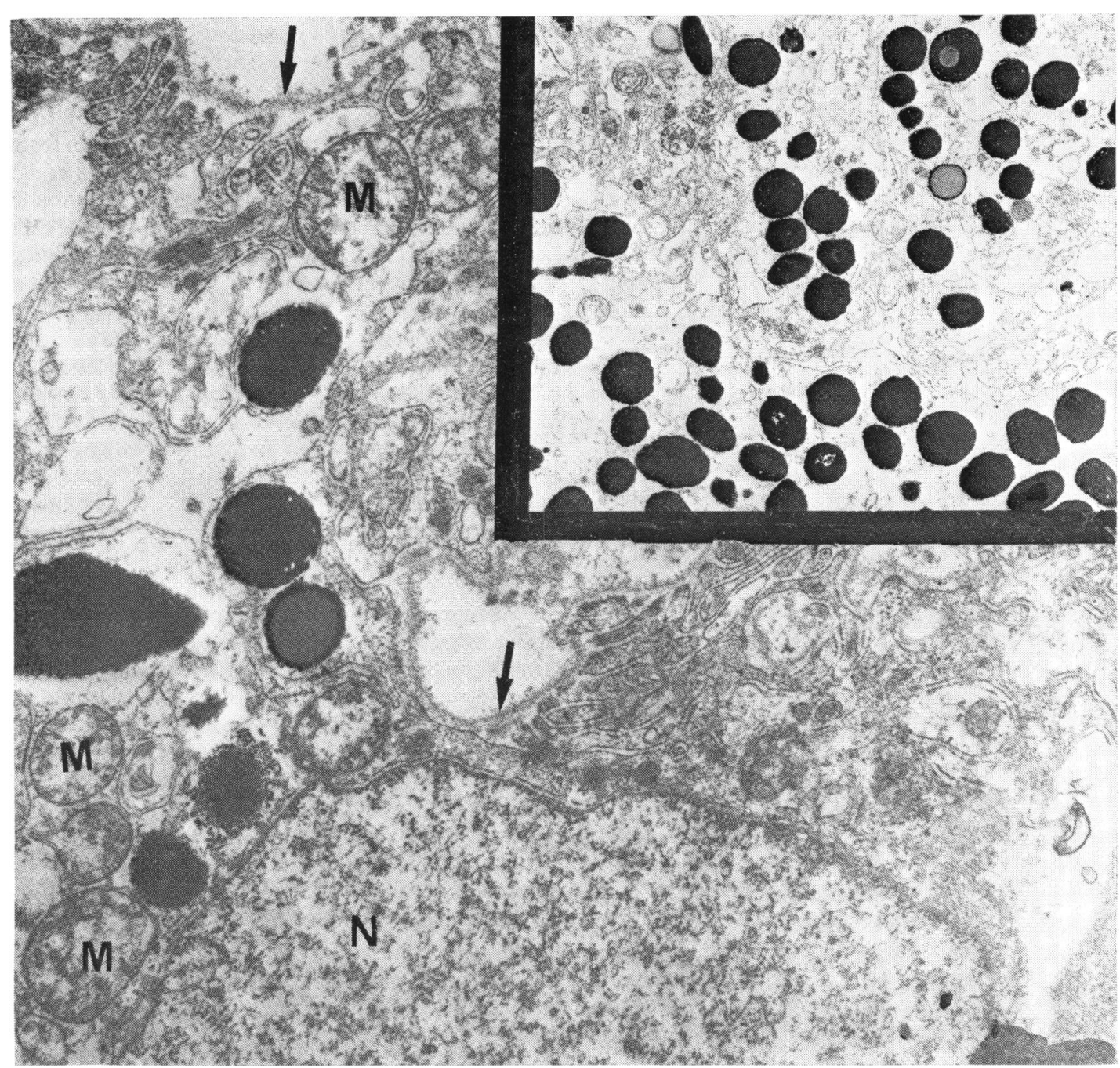

Fig. 5 Endothelial cell with melanin granules and prominent basement membrane (arrow). Mitochondrium (M). Nucleus $(\mathrm{N}) .(\times 13$ 500.) Inset: Pigment granules within cytoplasm of a macrophage. $(\times 6500)$.

aggravated pre-existing damage to the drainage area, resulting in a persistent increase in IOP.

We thank Mrs E. Neidhardt and Mrs B. Vozi for technical assistance.

\section{References}

1 Mitsui Y, Takagi Y. Nature of aqueous floaters due to sympaticomimetic mydriatics. Arch Ophthalmol 1961; 65: 626-31.

2 Kristensen P. Mydriasis-induced pigment liberation in the anterior chamber associated with acute rise in intraocular pressure in open-angle glaucoma. Acta Ophthalmol (Kbh) 1965; 43: 714-24.
3 Epstein DL, Bogner WP, Grant M. Phenylephrine provocative testing in the pigmentary dispersion syndrome. Am J Ophthalmol 1978; 85: 43-50.

4 Roth M, Epstein DL. Exfoliation syndrome. Am J Ophthalmol 1980; 89: 477-82.

5 Vrabec CF. The endothelium of the anterior chamber angle. In: Smelser GK, ed. The Structure of the Eye. New York: Academic Press, 1961: 311-24.

6 Fine BS, Yanoff M, Scheie HG. Pigmentary 'glaucoma'. A histologic study. Trans Am Acad Ophthalmol Otolaryngol 1974; 78: 314-25.

7 Hoffman F, Dumitrescu L, Hager H. Pigmentglaukom. Rasterelektronen-mikroskopische Befunde. Klin Monatsbl Augenheilkd 1975; 166: 609-13.

8 Rodrigues MM, Spaeth GL, Weinreb S, Sivalingam E. Spectrum of trabecular pigmentation in open-angle 
glaucoma. A clinico-pathologic study. Trans Am Acad Ophthalmol Otolaryngol 1976; 81: 258-76.

9 Richardson TM, Hutchinson BT. Grant WM. The outflow tract in pigmentary glaucoma. Arch Ophthalmol 1977; 95: 1015-25.

10 Rohen JW, Zypen E. The phagocytic activity of the trabecular meshwork endothelium. An electron microscopic study on the vervet (Cercopithecus aethiops). Albrecht von Graefes Arch Klin Ophthalmol 1968; 175: 143-60.

11 Ringvold A, Vegge T. Electron microscopy of the trabecular meshwork in eyes with exfoliation syndrome. Virchows Arch (Pathol Anat) 1971; 353: 110-27.

12 Lee WR. The study of the passage of particles through the endothelium of the outflow apparatus of the monkey eye by scanning and transmission electron microscopy. Trans Ophthalmol Soc UK 1971; 91 : 687-705.

13 Shabo al, Maxwell DS. Observations on the fate of blood in the anterior chamber. A light and electron microscopic study of the monkey trabecular meshwork. Am J Ophthal. mol 1972; 73: 25-36.

14 Grierson I, Lee WR. Further observations on the process of haemophagocytosis in the human outflow system. In: Spitznas M, ed. Current Research in Ophthalmic Electron Microscopy. Berlin: Springer, 1978: 49-64.

15 Grierson I, Chisholm IA. Clearance of debris from the iris through the drainage angle of the rabbit's eye. $\mathrm{Br} \mathrm{J}$ Ophthalmol 1978; 62: 694-704.

16 Rohen JW, Unger HH. Studies on the morphology and pathology of the trabecular meshwork in the human eye. Am J Ophthalmol 1958; 46: 802-13.

17 Tripathi RC. Aqueous outflow pathway in normal and glaucomatous eyes. Br J Ophthalmol 1972; 56: 157-74.

18 Grierson I, Lee WR. Erythrocyte phagocytosis in the human trabecular meshwork. Br J Ophthalmol 1973; 57: 400-15.

19 Yanoff M, Scheie HG. Melanomalytic glaucoma. Arch Ophthalmol 1970; 84: 471-3.

20 Flocks M, Littwin CS, Zimmerman LE. Phacolytic glaucoma. Arch Ophthalmol 1955; 54: 37-45.

21 Fenton RH, Zimmerman LE. Haemolytic glaucoma. An unusual cause of acute open-angle secondary glaucoma. Arch Ophthalmol 1963; 70: 236-9.

22 Iwamoto T, Witmer R, Landolt E. Light and electron microscopy in absolute glaucoma with pigment dispersion phenomenon and contusion angle deformity. $\mathrm{Am} J$ Ophthalmol 1971; 72: 420-34.

23 Rohen JW. Functional anatomy, physiology and pathology. In: Heilman K, Richardson KT, eds. Glaucoma: Conceptions of a Disease. Stuttgart: Thieme, 1978: 26-43.

24 Bill A. The drainage of aqueous humor. Invest Ophthalmol Visual Sci 1975; 14: 1-3. 